TRANSACTIONS OF THE

AMERICAN MATHEMATICAL SOCIETY

Volume 284. Number I. July 1984

\title{
PROPER HOLOMORPHIC MAPPINGS \\ THAT MUST BE RATIONAL
}

BY

STEVEN BELL

\begin{abstract}
Suppose $f: D_{1} \rightarrow D_{2}$ is a proper holomorphic mapping between bounded domains in $\mathbf{C}^{n}$. We shall prove that under certain circumstances $f$ must be a rational mapping, i.e., that the $n$ component functions $f_{i}$ of $f$ are rational functions.
\end{abstract}

THEOREM 1. Suppose $D_{1}$ is a bounded domain in $\mathbf{C}^{n}$ whose associated Bergman kernel function is a rational function and suppose $D_{2}$ is a bounded circular domain in $\mathbf{C}^{n}$ that contains the origin. Then any proper holomorphic mapping $f: D_{1} \rightarrow D_{2}$ must be rational.

The ball and the polydisc are examples of domains with rational kernel functions. Later in this note, we shall prove that Rudin's domains [3] that occur as proper images of the ball also have rational kernel functions.

The proof of the theorem uses the transformation formula for the Bergman kernel under proper holomorphic mappings proved in [1]. Let $K_{1}(z, w)$ and $K_{2}(z, w)$ denote the kernel functions associated to $D_{1}$ and $D_{2}$. The Remmert Proper Mapping Theorem asserts that $f$ is a branched cover of some finite order $m$. Let $F_{1}, F_{2}, \ldots, F_{m}$ denote the inverses to $f$ that are defined locally on $D_{2}$ minus the image of the branch locus under $f$. We shall write $u=\operatorname{det}\left[f^{\prime}\right]$ and $U_{k}=\operatorname{det}\left[F_{k}^{\prime}\right]$. The kernel functions transform according to

$$
u(z) K_{2}(f(z), w)=\sum_{k=1}^{m} K_{1}\left(z, F_{k}(w)\right) \overline{U_{k}(w)} .
$$

The theorem will follow from the transformation formula and two lemmas, which we now list. Let $w$ be a fixed point in $D_{2}$ and let $\partial^{\alpha}=\partial^{\alpha} / \partial w^{\alpha}$ denote the standard holomorphic differential operator of order $\alpha$. The first lemma concerns the linear functional $\Lambda$ on the space $H\left(D_{1}\right)$ of holomorphic functions in $L^{2}\left(D_{1}\right)$ given by

$$
\Lambda h=\partial^{\alpha}\left(\sum_{k=1}^{m} U_{k}\left(h \circ F_{k}\right)\right)(w) .
$$

Lemma 1 states that $\Lambda h$ only depends on finitely many derivatives of $h$ at the points in $f^{-1}(w)$.

Received by the editors October 3, 1983 and in revised form, November 10, 1983.

1980 Mathematics Subject Classification. Primary 32H99; Secondary 32H10.

(c) 1984 American Mathematical Society $0002-9947 / 84 \$ 1.00+\$ .25$ per page 
LEMMA 1. Let $\zeta_{1}, \zeta_{2}, \ldots, \zeta_{q}$ denote the points in $f^{-1}(w)$. There exist a positive integer $s$ and constants $c_{l, \alpha}$ such that

$$
\Lambda h=\sum_{l=1}^{q} \sum_{|\beta| \leqslant s} c_{l, \beta} \partial^{\beta} h\left(\zeta_{l}\right)
$$

for all $h$ in $H\left(D_{1}\right)$.

Note that $q \leqslant m$ and that Lemma 1 is only nontrivial in case $q<m$. To appreciate the meaning of Lemma 1 , it is instructive to consider the example $f(z)=z^{m}$ which is a proper self-map of the unit disc. In this case, if $w=x^{m} \neq 0$, then

$$
\sum_{k=1}^{m} U_{k}\left(h \circ F_{k}\right)=\left(m x^{m-1}\right)^{-1} \sum_{k=1}^{m} \eta^{-k(m-1)} h\left(x \eta^{k}\right)
$$

where $\eta=e^{2 \pi i / m}$. If $w=0$, then this sum is equal to $h^{(m-1)}(0) /(m-1) !$. The proof of Lemma 1 is deferred.

The second lemma is a well-known result about circular domains that contain the origin. A proof of Lemma 2 can be found in [2].

LEMMA 2. If $K(z, w)$ is the Bergman kernel function associated to a bounded circular domain in $\mathbf{C}^{n}$ that contains the origin, then $K(z, 0)$ is a constant function of $z$. Furthermore, there is an $n \times n$ matrix of constants $\left(a_{i j}\right)$ such that the ith coordinate function $z_{i}$ can be written

$$
z_{i}=\sum_{j=1}^{m} a_{i j} K^{j}(z, 0) .
$$

Here we have used the shorthand notation $K^{j}(z, w)=\left(\partial / \partial \bar{w}_{j}\right) K(z, w)$.

We shall now prove the theorem assuming the truth of the lemmas. Let $\left\{\zeta_{1}, \ldots, \zeta_{q}\right\}=f^{-1}(0)$. Lemma 1 implies that there are constants $c_{l, \beta}^{j}$ such that

$$
\left(\frac{\partial}{\partial w_{j}} \sum_{k=1}^{m} U_{k}\left(h \circ F_{k}\right)\right)(0)=\sum_{l=1}^{q} \sum_{|\beta| \leqslant s} c_{l, \beta}^{j} \partial^{\beta} h\left(\zeta_{l}\right)
$$

for all $h$ in $H\left(D_{1}\right)$. Hence, if we differentiate the transformation formula with respect to $\bar{w}_{j}$ and set $w=0$, we obtain the formula

$$
u(z) K_{2}^{j}(f(z), 0)=\sum_{l=1}^{q} \sum_{|\beta| \leqslant s} \bar{c}_{l, \beta}^{j} K_{1}^{\bar{\beta}}\left(z, \zeta_{l}\right) .
$$

Here we have written $K_{1}^{\bar{\beta}}(z, w)$ for $\left(\partial^{\beta} / \partial \bar{w}^{\beta}\right) K_{1}(z, w)$. This formula yields that $u(z) K_{2}^{j}(f(z), 0)$ is a rational function of $z$. Similarly, $u(z) K_{2}(f(z), 0)$ is a rational function. Finally, Lemma 2 can be used to deduce that $f_{i}(z)$ is a constant times

$$
\left(\sum_{j=1}^{n} a_{i j} u(z) K_{2}^{j}(f(z), 0)\right) /\left(u(z) K_{2}(f(z), 0)\right)
$$

which we know to be a rational function. Theorem 1 is reduced to proving Lemma 1. 
Proof of Lemma 1. Let $\Theta$ be a $C^{\infty}$ radially symmetric function that is supported in the unit ball of $\mathbf{C}^{n}$ with $\int \Theta=1$. Let $\Theta_{\varepsilon}(z)=\varepsilon^{-2 n} \Theta((z-w) / \varepsilon)$ and $\Theta_{\varepsilon}^{\alpha}=$ $(-1)^{|\alpha|} \bar{\partial}^{\alpha} \theta_{\varepsilon}$. Note that $\Theta_{\varepsilon}^{\alpha}$ is supported in the ball $B_{\varepsilon}=B(w ; \varepsilon)$ of radius $\varepsilon$ centered at $w$. Let $\varepsilon>0$ be small enough that $B_{\varepsilon} \subset D_{2}$. Now

$$
\left(\partial^{\alpha} \sum_{k=1}^{m} U_{k}\left(h \circ F_{k}\right)\right)(w)=\int_{D_{2}}\left(\sum_{k=1}^{m} U_{k}\left(h \circ F_{k}\right)\right) \overline{\Theta_{\varepsilon}^{\alpha}}=\int_{D_{1}} h \overline{u\left(\Theta_{\varepsilon}^{\alpha} \circ f\right)} .
$$

Using this identity and the fact proved in [1] that

$$
\left\|u\left(\Theta_{\varepsilon}^{\alpha} \circ f\right)\right\|_{L^{2}\left(D_{1}\right)}=m^{1 / 2}\left\|\Theta_{\varepsilon}^{\alpha}\right\|_{L^{2}\left(D_{2}\right)}=(\text { constant }) \varepsilon^{-n-|\alpha|},
$$

we obtain the inequality,

$$
|\Lambda h| \leqslant(\text { constant }) \varepsilon^{-n-|\alpha|}\|h\|_{L^{2}\left(f^{-1}\left(B_{\varepsilon}\right)\right)} .
$$

The Nullstellensatz implies that there are neighborhoods $N_{1}, N_{2}, \ldots, N_{q}$ of $\zeta_{1}, \zeta_{2}, \ldots, \zeta_{q}$, respectively, and positive integers $m_{1}, m_{2}, \ldots, m_{q}$ such that

$$
\left|z-\zeta_{l}\right|^{m_{l}} \leqslant|f(z)-w| \text { for } z \text { in } N_{l} \text {. }
$$

Hence $f^{-1}\left(B_{\varepsilon}\right) \subset \cup q_{=1} B\left(\zeta_{l} ; \varepsilon^{1 / m_{l}}\right)$ for small $\varepsilon>0$. Therefore, if $h$ vanishes to order $s$ at each of the points $\zeta_{1}, \ldots, \zeta_{q}$, then

$$
\|h\|_{L^{2}\left(f^{-1}\left(B_{\varepsilon}\right)\right)} \leqslant(\text { constant }) \sum_{l=1}^{q} \varepsilon^{(s+n) /\left(m_{l}\right)} .
$$

We now have that $|\Lambda h| \leqslant($ constant $) \sum q_{=1} \varepsilon^{\left(t_{l}\right)}$ where $t_{l}=(s+n) /\left(m_{l}\right)-n-|\alpha|$. Thus, by letting $\varepsilon \rightarrow 0$, we see that $\Lambda h=0$ whenever $h$ vanishes to order $s$ at each of the points $\zeta_{1}, \ldots, \zeta_{q}$ with $s>\operatorname{Max}\left\{m_{l}(n+|\alpha|)-n\right\}$. This finishes the proof of Lemma 1.

REMARK. If $\Omega$ is a bounded domain in $\mathbf{C}^{n}$, let $\Phi_{\Omega}$ denote the complex linear span of the set $\left\{\left(\partial^{\alpha} / \partial \bar{w}^{\alpha}\right) K(z, w): w \in \Omega ;|\alpha| \geqslant 0\right\}$ where $K(z, w)$ is the Bergman kernel for $\Omega$. During the course of the proof of Theorem 1 , the following fact was also shown to be true.

THEOREM 2. Suppose $f: \Omega \rightarrow D$ is a proper holomorphic mapping of a bounded domain $\Omega$ in $\mathbf{C}^{n}$ onto a bounded circular domain $D$ in $\mathbf{C}^{n}$ that contains the origin. Then the components of $f$ are functions of the form $s_{i} / s$ where $s_{i}$ and $s$ are elements of $\Phi_{\Omega}$.

Theorem 2 generalizes a familiar formula from one complex variable theory. If $f$ : $\Omega \rightarrow \Delta$ is the Riemann mapping function of a bounded simply connected domain $\Omega$ in $\mathbf{C}$ onto the unit disc $\Delta$ such that $f(a)=0$ and $f^{\prime}(a)>0$, then

$$
f(z)=\pi^{-3 / 2} K(a, a)^{-1 / 2}\left[\frac{K_{\overline{\bar{w}^{\prime}}}(z, a)}{K(z, a)}-\frac{K_{\overline{\mathrm{w}}}(a, a)}{K(a, a)}\right]
$$

where $K(z, w)$ denotes the Bergman kernel for $\Omega$ and $K_{\bar{w}}(z, w)=d K(z, w) / d \bar{w}$.

Rudin's domains. In [3], Rudin studied a class of bounded domains in $\mathbf{C}^{n}$ that arise as images of the unit ball $B$ in $\mathbf{C}^{n}$ under a proper holomorphic mapping. We shall now show that Rudin's domains have rational Bergman kernel functions. 
Let $G$ be a finite subgroup of the group of unitary transformations of $\mathbf{C}^{n}$. Suppose further that $G$ is generated by the reflections that it contains. The Rudin domain $R_{G}$ associated to the unitary reflection group $G$ is defined as follows. The ChevalleyHilbert Theorem (see [3]) asserts that there are exactly $n$ homogeneous $G$-invariant polynomials $p_{1}, p_{2}, \ldots, p_{n}$ which generate the ring of all $G$-invariant polynomials. Define a mapping $P: \mathbf{C}^{n} \rightarrow \mathbf{C}^{n}$ via $P(z)=\left(p_{1}(z), \ldots, p_{n}(z)\right)$. Ruding proved that $P$ maps $B$ properly onto $P(B)$. The domain $R_{G}$ is defined to be $P(B)$.

Let $K_{B}(z, w)$ denote the Bergman kernel for $B$ and $K_{G}(z, w)$ the kernel for $R_{G}$. Since $P$ is a branched cover of some finite order $m$, we may let $F_{1}, \ldots, F_{m}$ denote the local inverses to $P$ that are defined on a dense open subset of $R_{G}$. The number $m$ is also equal to the order of the group $G$. We shall write $u=\operatorname{det}\left[P^{\prime}\right]$ and $U_{k}=\operatorname{det}\left[F_{k}^{\prime}\right]$ as before. The kernel functions transform under $P$ according to

$$
u(z) K_{G}(P(z), w)=\sum_{k=1}^{m} K_{B}\left(z, F_{k}(w)\right) \overline{U_{k}(w)} .
$$

Set $z=F_{j}(x)$ in this formula and notice that $P\left(F_{j}(x)\right)=x$ to obtain

$$
K_{G}(x, w)=\sum_{k=1}^{m} U_{j}(x) K_{B}\left(F_{j}(x), F_{k}(w)\right) \overline{U_{k}(w)} .
$$

Now sum these equations over $j$ to get the useful formula

$$
m K_{G}(z, w)=\sum_{j=1}^{m} \sum_{k=1}^{m} U_{j}(z) K_{B}\left(F_{j}(z), F_{k}(w)\right) \overline{U_{k}(w)} .
$$

We shall use formula (1.1) together with the following lemma to prove that $K_{G}(z, w)$ is rational.

Lemma 3. Suppose $D$ is a bounded circular domain that contains the origin. The kernel function $K_{D}(z, w)$ associated to $D$ is rational if and only if there is a polynomial $A(z, w)$ on $\mathbf{C}^{2 n}$ and a positive integer $N$ such that

$$
\int_{D}\left(\int_{D} K_{D}(z, w) A(z, \bar{w}) \bar{z}^{\alpha} w^{\beta} d V_{w}\right) d V_{z}=0
$$

for all multi-indices $\alpha$ and $\beta$ with $|\alpha|+|\beta|>N$.

This lemma follows easily from the fact that a function $h$ in $H(D)$ is orthogonal to $z^{\alpha}$ for each $\alpha$ with $|\alpha|=N$ if and only if $\left(\partial^{\alpha} h / \partial z^{\alpha}\right)(0)=0$ for all $\alpha$ with $|\alpha|=N$. It should be pointed out that the monomials $z^{\alpha}$ do not, in general, form an orthogonal set in $H(D)$. However, $\int_{D} z^{\alpha} \bar{z}^{\beta}=0$ whenever $|\alpha| \neq|\beta|$.

Write $G=\left\{g_{1}, \ldots, g_{m}\right\}$. Let $Q(z, \bar{w})^{-1}=\prod_{j=1}^{m} \prod_{k=1}^{m} K_{B}\left(g_{j}(z), g_{k}(w)\right)$. Notice that $Q(z, w)$ is a polynomial on $\mathbf{C}^{2 n}$ that is $G \times \bar{G}$ invariant. It can be shown that the set of $G \times \bar{G}$ invariant polynomials is generated by

$$
p_{1}(z), p_{2}(z), \ldots, p_{n}(z), \overline{p_{1}(\bar{w})}, \overline{p_{2}(\bar{w})}, \ldots, \overline{p_{n}(\bar{w})}
$$

where $(z, w)$ is a point in $\mathbf{C}^{2 n}=\mathbf{C}^{n} \times \mathbf{C}^{n}$. Hence, there is a polynomial $A(z, w)$ on $\mathrm{C}^{2 n}$ such that $Q(z, \bar{w})=A(P(z), \overline{P(w)})$. 
Since $R_{G}$ is a bounded circular domain that contains the origin, Lemma 3 applies. Notice that formula (1.1) yields that

$$
\begin{aligned}
& \int_{R_{G}}\left(\int_{R_{G}} K_{G}(z, w) A(z, \bar{w}) \bar{z}^{\alpha} w^{\beta} d V_{w^{\prime}}\right) d V_{z} \\
& \quad=\frac{1}{m} \int_{R_{G}}\left(\int_{B} \sum_{j=1}^{m} U_{j}(z) K_{B}\left(F_{j}(z), \zeta\right) u(\zeta) A(z, \overline{P(\zeta)}) \bar{z}^{\alpha} P(\zeta)^{\beta} d V_{\zeta}\right) d V_{z} \\
& \quad=\frac{1}{m} \int_{B}\left(\int_{B} K_{B}(x, \zeta) \overline{u(x)} A\left(p(x), \overline{P(\zeta))} u(\zeta) \overline{P(x)}{ }^{\alpha} P(\zeta)^{\beta} d V_{\zeta}\right) d V_{x} .\right.
\end{aligned}
$$

Now $K_{B}(x, \zeta) A\left(P(x), \overline{P(\zeta))}=K_{B}(x, \zeta) Q(x, \bar{\zeta})\right.$ is a polynomial in $x$ and $\bar{\zeta}$. Hence, if $|\alpha|+|\beta|$ is sufficiently large, then $\overline{u(x) P(x)^{\alpha}} P(\zeta)^{\beta} u(\zeta)$ is a homogeneous polynomial in $\bar{x}$ and $\zeta$ of high degree, and the integrals above vanish. This completes the proof that $K_{G}(z, w)$ is rational.

REMARK. By combining Rudin's results with results of the present work, we are able to obtain

THEOREM 3. Suppose $f: B \rightarrow D$ is a proper holomorphic mapping of the ball in $\mathbf{C}^{n}$ onto a circular domain $D$ in $C^{n}$ which contains the origin. Then $f=R \circ P \circ \Phi$ where $P$ is the Rudin polynomial mapping associated to $f, R$ is a rational mapping which is biholomorphic from $P(B)$ onto $D$, and $\Phi$ is an automorphism of the ball.

\section{REFERENCES}

1. S. Bell, The Bergman kernel function and proper holomorphic mappings, Trans. Amer. Math. Soc. 270 (1982), 685-691.

2. $\ldots$, Proper holomorphic mappings between circular domains, Comment. Math. Helv. 57 (1982), 532-538.

3. W. Rudin, Proper holomorphic maps and finite reflection groups, Indiana Math. J. 31 (1982), 701-720.

Department of Mathematics, Princeton University, Princeton, New Jersey 08544 